\title{
Competência Cultural na Intervenção com Imigrantes: Uma Análise Comparativa entre Profissionais da Saúde, da Área Social e Polícias
}

\section{Cultural Competence in Intervention with Immigrants: A Comparative Analysis Between Health Professionals, Social Workers and Police Officers}

\author{
Mariana GONÇALVES $\triangle^{1}$, Marlene MATOS ${ }^{1}$ \\ Acta Med Port 2016 Oct;29(10):629-638 - http://dx.doi.org/10.20344/amp.7121
}

\section{RESUMO}

Introdução: A diversidade cultural coloca exigências acrescidas no atendimento a populações multiculturais, pelo que o desenvolvimento de competências culturais por parte dos profissionais de ajuda é, atualmente, uma preocupação ao nível das práticas institucionais.

Material e Métodos: Este estudo avaliou a perceção sobre competência cultural dos profissionais de ajuda de três áreas distintas: serviços de saúde, serviços sociais e órgãos de polícia criminal. Através de um questionário online analisámos a perceção sobre a competência cultural, avaliada em quatro dimensões: consciência cultural, conhecimento cultural, aptidões técnicas, apoio organizacional. Os 610 participantes eram maioritariamente do sexo feminino (58\%), com média de idade de 39,74 anos, desenvolvia atividade na área social $(37 \%)$, da saúde (33\%) ou nas polícias (30\%).

Resultados: Os profissionais revelaram, em geral, uma perceção positiva da sua competência cultural. Aqueles que beneficiaram de mais experiências formativas sobre o tema e possuíam mais tempo de serviço percecionaram-se, de forma significativa, como mais competentes culturalmente. Foram encontradas diferenças significativas entre os profissionais das diferentes áreas: os profissionais de saúde percecionavam-se como mais eficazes ao nível das aptidões técnicas, os profissionais da área social ao nível do conhecimento cultural e os polícias ao nível da consciência cultural. Os profissionais de saúde foram os que revelaram uma menor perceção no que respeita ao apoio organizacional.

Discussão: Apesar da perceção positiva que os técnicos têm acerca da sua consciência e conhecimento sobre os valores, normas e costumes das comunidades imigrantes com as quais trabalham, percecionam a sua aptidão técnica como menos positiva, mostrando dificuldade na aplicação prática desses conhecimentos.

Conclusão: A competência cultural tem implicações para uma boa prática profissional no atendimento de populações multiculturais, sendo urgente investir no desenvolvimento de intervenções culturalmente competentes, de forma a garantir atuações mais eficazes nomeadamente nos hospitais e nos centros de saúde.

Palavras-chave: Atitude do Pessoal de Saúde; Competência Cultural; Conhecimentos, Atitudes e Prática em Saúde; Emigrantes e Imigrantes.

\section{ABSTRACT}

Introduction: Cultural diversity places increased demands on services to multicultural populations, so the development of cultural competence by help professionals is currently a concern in institutional practices.

Material and Methods: This study evaluated the perception of cultural competence of help professional of three distinct areas: health services, social services and criminal police. Through an online questionnaire, we questioned the perception of cultural competence, at four dimensions: cultural awareness, cultural knowledge, technical skills, and organizational support. There were 610 participants, mostly female $(58 \%)$, with a mean age of 39.74 years, developing activity in the social area $(37 \%)$, health $(33 \%)$ or the police $(30 \%)$.

Results: The professionals showed, in general, a positive perception of their cultural competence. Those who had formative experiences on the subject and had more time service, perceived themselves, significantly, as more culturally competent. Significant differences were found between professionals from different areas: health professionals were more effective in terms of technical skills, the social workers at the level of cultural knowledge and polices at the level of cultural awareness. Health professionals were the ones that showed a lower perception at the level of organizational support.

Discussion: Despite the positive perception that technicians have about their awareness and knowledge of the values, norms and customs of immigrant communities, they realize technical aptitude as less positive, showing difficulty in practical application of their knowledge.

Conclusions: Cultural competence has implications for good professional practice in serving multicultural populations, being urgent to invest in the development of culturally competent interventions to ensure more effective services, namely in hospitals and health centres.

Keywords: Attitude of Health Personnel; Cultural Competency; Emigrants and Immigrants; Health Knowledge, Attitudes, Practice.

\section{INTRODUÇÃO}

A diversidade multicultural tem levado os profissionais de diversas áreas, a nível mundial, a refletir sobre a necessidade de desenvolver serviços culturalmente competen-

tes, no atendimento, no acompanhamento e na intervenção junto de populações culturalmente diferenciadas. Esta temática tem estado presente também na dinâmica das

1. Escola de Psicologia. Universidade do Minho. Braga. Portugal.

$\square$ Autor correspondente: Mariana Gonçalves. marianavgcosta@gmail.com

Recebido: 24 de outubro de 2015 - Aceite: 21 de março de 2016 | Copyright $\odot$ Ordem dos Médicos 2016 
organizações e nos programas de ação de âmbito internacional que colocam a competência cultural como uma prioridade na sua missão organizacional (e.g., National Center for Cultural Competence, EUA; Department of Health and Human Services, EUA; The Cultural Competency Training Program, Canadá), bem como a necessidade de inclusão destas temáticas nos curricula académicos. ${ }^{1}$

\section{Competência cultural: Definição e dimensões de ava- liação}

O conceito de competência cultural tem suscitado debate na literatura, não só ao nível da sua definição, mas também enquanto conceito, modelo ou teoria. ${ }^{2}$ Muitos são os trabalhos científicos que problematizam a sua definição, ${ }^{2-5}$ a sua mensuração $0^{6,7}$ e os modelos concetuais subjacentes. 8,9

Para a Antropologia, a cultura não pode ser vista como homogénea ou estática. Os antropólogos enfatizam que a cultura não é uma variável única, compreendendo antes múltiplas variáveis, afetando todos os aspetos da experiência. A cultura é inseparável das condições económicas, políticas, religiosas, psicológicas e biológicas. ${ }^{10}$ A Antropologia tem sido crítica em relação ao conceito de competência cultural, na medida em que este sugere que a cultura pode ser reduzida a uma entidade estática e a uma capacidade técnica para a qual os profissionais podem ser treinados. ${ }^{10,11}$ Essa questão constituir-se-á um problema em função da forma como a cultura é definida em profissões como a Medicina, o que contrasta notavelmente com seu uso atual na Antropologia - o campo de onde é originário o conceito de cultura. ${ }^{10-12}$ Cultura é, muitas vezes, entendida como sinónimo de etnia, nacionalidade e língua, ${ }^{10,13}$ sendo essa visão redutora. Em vez disso, os antropólogos entendem a cultura como um conjunto de "símbolos compartilhados e significados que as pessoas criam no processo de interação social". ${ }^{13}$

A discussão em torno deste fenómeno é, apesar de tudo, relativamente recente, tendo surgido na literatura a partir da década de 80 , nas áreas social ${ }^{2,14,15}$ e da psicoterapia, ${ }^{16,17}$ expandindo-se posteriormente às áreas da enfermagem, educação e literatura médica. ${ }^{18-20}$

Durante este breve percurso, foram avançadas várias definições relacionadas com as diferentes áreas de atuação. Uma das mais citadas e robustas surgiu na área social e foi desenvolvida por Cross: "a competência cultural é um conjunto de comportamentos, atitudes e políticas congruentes que atuam interactivamente num sistema, instituição ou grupo de profissionais, e permite que esse sistema, instituição ou grupo de profissionais trabalhem efetivamente em situações multiculturais". ${ }^{14}$

Já na área da saúde, a competência cultural é entendida como um processo contínuo em que o prestador de cuidados de saúde se esforça continuamente para alcançar a capacidade de trabalhar de forma eficaz e congruente com o contexto cultural do cliente, ${ }^{18}$ demonstrando compreensão e respeito pelas diferenças ao nível das crenças e dos comportamentos relacionados com a saúde, reconhecendo as variações que ocorrem entre os diferentes grupos e estando capaz de ajustar a sua prática para fornecer intervenções adaptadas culturalmente. .,18 $^{-18}$

Neste sentido, qualquer que seja a área de atuação profissional, o processo de desenvolvimento de competências culturais é, portanto, dinâmico e envolve fatores contextuais, desenvolvimentais e de crescimento pessoal, resultando na compreensão profissional e na capacidade de prestar serviços adequados aos indivíduos que olham, pensam e se comportam de maneira diferente da cultura dominante. ${ }^{7}$ A competência cultural, como um todo, envolve um conjunto de constructos, consensualmente agrupados em diferentes dimensões: cognitiva (atitudes/crenças e conhecimento culturais), comportamental (competência prática) e organizacional. ${ }^{21,22}$

Recentemente foi proposto um modelo compreensivo do fenómeno, ${ }^{5}$ baseado numa revisão sistemática da literatura, que integra os quatro constructos mais comummente identificados nesse âmbito: consciência cultural (apreciação e compreensão da cultura de outras pessoas, reconhecimento dos próprios preconceitos em relação a outras culturas e exame crítico em relação a posições privilegiadas na sociedade); conhecimento cultural (familiaridade com as características, história, valores, crenças e comportamentos de outras culturas); competência ou aptidão prática (aptidões necessárias para ajustar a prática profissional de forma a atender às necessidades das populações multiculturais) e, finalmente, o apoio organizacional (implementação de práticas individuais e organizacionais que promovam a capacidade dos profissionais para intervir de forma culturalmente adequada). ${ }^{5}$ Segundo os autores da proposta, o desenvolvimento da competência cultural no seu todo é um processo contínuo e interativo que pressupõe que, enquanto prestadores de serviços, os profissionais estejam familiarizados e trabalhem confortavelmente tendo em conta as especificidades dos grupos culturais com os quais lidam. Para isso contribuem fatores como a formação prévia em competência cultural, o tempo de serviço e as atitudes face à temática. Num estudo realizado com profissionais de reabilitação, aqueles que verbalizaram sentir-se culturalmente mais competentes pontuaram mais alto em todas as dimensões do constructo (consciência/conhecimento, aptidões práticas e apoio organizacional). ${ }^{5}$

Porém, são escassos os estudos que esclarecem em que medida os profissionais das mais diversas áreas (saúde, educação, social, criminal) se sentem capazes de intervir, e o são efetivamente, quando confrontados com populações provenientes de meios culturalmente distintos.

\section{Competência cultural: Qual é o seu impacto nos profis- sionais de ajuda?}

Alguns estudos realizados com enfermeiros têm evidenciado a importância das intervenções culturalmente competentes com populações multiculturais, ao proporcionar uma melhoria nos cuidados prestados ao nível da saúde da mulher e redução das disparidades comparativamente com a cultura dominante, ${ }^{23}$ com implicações positivas nos 
processos de comunicação, no fortalecimento da relação de confiança e na adesão terapêutica. ${ }^{24}$ Ainda na área da saúde, estudos com médicos revelaram que o conhecimento acerca das características epidemiológicas de grupos específicos, a sensibilidade para atender a diferentes valores culturais e o conhecimento de outras línguas permite a esses profissionais o desenvolvimento de práticas culturalmente adaptadas, ${ }^{25}$ com implicações efetivas, por exemplo, ao nível da adesão terapêutica na diabetes. ${ }^{26} \mathrm{~A}$ reforçar igualmente a importância das intervenções culturalmente competentes com populações multiculturais, uma revisão sistemática da literatura que integrou resultados de catorze estudos sobre os processos de comunicação entre médicos e doentes de grupos culturalmente diferenciados (e.g., latinos, hispânicos, turcos, marroquinos, aborígenes), concluiu que os médicos se comportavam de modo menos afetivo quando interagiam com doentes de minorias étnicas em comparação com doentes caucasianos. Os doentes de minorias étnicas, por sua vez, também interagiam menos com os médicos do que os caucasianos, eram verbalmente menos expressivos e menos assertivos. ${ }^{27}$ Um outro estudo, com doentes que sobreviveram a um diagnóstico de cancro, concluiu que vários anos após o diagnóstico inicial, os doentes de minorias étnicas estavam mais propensos a ter necessidades não atendidas, relacionadas com a informação disponibilizada e na obtenção de ajuda para problemas físicos. ${ }^{28}$

$\mathrm{Na}$ intervenção dos serviços junto de vítimas de crime, os estudos focam-se sobretudo nas crenças e nas atitudes que influenciam a prática profissional, nomeadamente no que respeita à violência doméstica, quer nos profissionais de ajuda, ${ }^{29,30}$ quer nos polícias, ${ }^{30-32}$ bem como nas implicações que as mesmas poderão ter na vítima, sobretudo quando a mesma enfrenta exigências já acrescidas por ser de outra nacionalidade ou cultura diferente da dominante. ${ }^{33}$

Quando se trata de mulheres vítimas imigrantes, para além da vitimação, enfrentam, no país que as acolhe, um conjunto de exigências acrescidas e/ou barreiras à procura de ajuda, ${ }^{34}$ relacionadas com a língua e estatuto de residência, ${ }^{35}$ com a dependência económica, medo de pobreza e receio do sistema de justiça, com o desconhecimento dos seus direitos legais e opções/recursos existentes institucionalmente e na comunidade. ${ }^{36}$ Apesar dessas barreiras, a procura de ajuda tende a ocorrer sobretudo em situações de maior gravidade, percecionadas pela vítima como colocando a sua vida em perigo, ${ }^{37,38}$ e sobretudo junto dos profissionais de saúde, de serviço social e/ou polícias. ${ }^{39-41}$ No entanto, continuam a ser visíveis, em alguns destes serviços, estereótipos, racismo e pressão para a aculturação das vítimas à cultura dominante, ${ }^{42}$ quer pela dificuldade dos técnicos em abordar a temática da violência, à luz das necessidades específicas e da cultura da vítima em questão, quer pelo facto das próprias instituições demonstrarem alguma dificuldade em reconhecer e adequar o seu funcionamento às especificidades das culturas comunitárias diferenciadas. ${ }^{40}$ Alguns estudos com populações pertencentes a minorias étnicas têm demonstrado que as vítimas avaliam como mais eficazes, acolhedores e apoiantes, os serviços sensíveis às especificidades de cada comunidade, onde as necessidades e a origem cultural das vítimas, nomeadamente no que respeita, por exemplo, à alimentação, são tidas em consideração nas interações e nas intervenções levadas a cabo. ${ }^{43}$

\section{Competência cultural: A situação em Portugal}

Apesar do decréscimo progressivo do número de residentes estrangeiros em Portugal, segundo o relatório Imigração, Fronteiras e Asilo (2014), Portugal continua a ser um país pautado pela diversidade cultural, integrando comunidades de várias origens étnicas (e.g., brasileira, cabo-verdiana, angolana, europa de leste, chinesa, ciganos). Esta diversidade acarreta exigências acrescidas aos profissionais das mais diversas áreas, exigindo que os serviços sejam prestados atendendo às necessidades específicas de cada um.

A nível nacional, a comunidade científica tem demonstrado algum interesse, ainda que insuficiente, quer em torno da discussão teórica do fenómeno, nomeadamente a necessidade de uma abordagem cultural à vitimação, ${ }^{44}$ quer em torno das competências culturais necessárias no atendimento a vítimas de crime. ${ }^{40,45} \mathrm{~A}$ nível empírico são conhecidos dois estudos em Portugal, na área da antropologia, ambos de cariz qualitativo, relacionados com a construção da competência cultural junto de profissionais de saúde mental e de enfermeiros. ${ }^{46,47}$ Segundo os autores, a construção da competência cultural, nos serviços de saúde mental, implica uma reflexão crítica sobre os contextos sociais e políticos das comunidades atendidas, bem como sobre os instrumentos e procedimentos de intervenção, de forma a evitar a homogeneização de experiências e vivências dos programas terapêuticos para populações culturalmente diferenciadas. ${ }^{46}$ Reis et $a{ }^{47}$ defendem que, nos enfermeiros, as competências culturais se constroem de uma forma processual no contexto dos cuidados. O processo inicia-se nas interações com as populações multiculturais, onde os enfermeiros têm a possibilidade de identificar as áreas deficitárias ao nível dos conhecimentos e habilidades culturais, tomando consciência do património cultural dos utentes.

No que respeita às políticas nacionais, apesar de estar já patente no atual Plano Estratégico para as Migrações ${ }^{48}$ (2015 - 2020) alguma preocupação em torno das necessidades das populações migrantes, não é ainda abordada abertamente a questão das intervenções culturalmente competentes. No entanto, a recente inclusão de medidas que pretendem, por exemplo, a promoção da formação dos profissionais de saúde acerca das necessidades dos imigrantes, é um avanço positivo nesse sentido.

De referir, ainda, que segundo o Migration Integration Police Index, ${ }^{49}$ apesar de Portugal ser o segundo melhor país (de um total de 38) a integrar imigrantes, a área da saúde é aquela em que a pontuação obtida é a mais baixa. Os responsáveis pela elaboração desse índice avançam, ainda, duas recomendações fundamentais para Portugal: 
investir nas competências multiculturais dos prestadores de serviços públicos para melhorar o acesso a serviços de melhor qualidade; sensibilizar os prestadores de serviços de saúde para progredir na capacidade de resposta às necessidades específicas de saúde dos migrantes a residir em Portugal.

\section{O presente estudo: Objetivos}

Este estudo teve como principal objetivo, através de uma análise comparativa, avaliar a perceção sobre competência cultural dos profissionais que exerciam funções em três áreas distintas: saúde, social e criminal.

A escolha desses três grupos de profissionais esteve relacionada com o facto de se tratar de técnicos de ajuda, que atuam na linha da frente, em serviços que funcionam muitas vezes $24 \mathrm{~h}$ e que, no âmbito das suas profissões, são por exemplo confrontados com imigrantes vítimas de crime (e.g., violência doméstica, tráfico humano, crimes sexuais, imigração e emprego ilegal). Estes processos de ajuda tornam necessário, por um lado, a sensibilidade para reconhecer os sinais de vitimação e, por outro lado, a capacidade de orientar o/a utente na procura de ajuda e no seu encaminhamento.

De forma mais detalhada, pretendemos captar a sua perceção acerca da capacidade para interagir e intervir com populações multiculturais. Adicionalmente, procedeu-se a uma análise comparativa entre alguns grupos de profissionais, no que respeita à sua perceção nas quatro dimensões da competência cultural, procurando identificar as dificuldades sentidas por esses grupos no atendimento a populações multiculturais (imigrantes e/ou minorias étnicas).

Para além de alertar e conscientizar para a necessidade da competência cultural nas profissões de ajuda, pretendemos fazer um diagnóstico de necessidades nessas áreas de atuação. Isso informaria sobre novas formas de potenciar o desenvolvimento de serviços de melhor qualidade, nomeadamente as necessidades de se investir na formação dos profissionais ao nível da sua competência cultural e na adoção de abordagens culturalmente adequadas nas instituições de atendimento.

\section{MATERIAL E MÉTODOS}

Para a realização deste estudo, foi enviado aos profissionais de ajuda o Questionário de Avaliação de Competências Culturais (QACC) que avaliava os seguintes parâmetros: características sociodemográficas (e.g., idade, sexo, nacionalidade); experiência profissional (e.g., tempo de serviço, experiência com populações multiculturais), formação prévia em competência cultural; avaliação do sucesso ao nível da interação e intervenção com populações multiculturais (numa escala de Likert variável entre um (muito insucesso) a seis (muito sucesso); perceção da competência cultural. O questionário foi traduzido, adaptado e validado para a população portuguesa do original - Cultural competence assessment instrument (CCAI) ${ }^{7}$ - depois da autorização dos autores. Constituído por 16 itens, este instrumento avalia a competência cultural a partir de quatro dimensões distintas: consciência cultural (três itens); conhecimento cultural (seis itens) (familiaridade com as características, história, valores, crenças e comportamentos de outras culturas), aptidões técnicas (três itens) (aptidões necessárias para ajustar a prática profissional de forma a atender às necessidades das populações multiculturais) e apoio organizacional (quatro itens) (implementação de práticas individuais e organizacionais que promovam a capacidade dos profissionais para intervir de forma culturalmente adequada). Foi realizada uma análise fatorial confirmatória para a validação do instrumento, com bons índices de ajustamento.

O questionário foi construído numa versão online e disseminado por várias instituições em Portugal: a) centros de saúde e hospitais, através das comissões de ética e por snowballing; b) centros locais de atendimento ao imigrante (CLAII); c) instituições de apoio à vítima (instituições particulares de solidariedade social (IPSS), organizações não governamentais (ONGs), instituições de acolhimento para vítimas de violência doméstica; d) órgãos de polícia criminal (OPC's) de proximidade (PSP e GNR). A recolha decorreu de fevereiro a maio de 2015.

O link para o questionário era acompanhado por uma nota prévia com o enquadramento e os objetivos do estudo, esclarecendo ainda as questões éticas no preenchimento do questionário (e.g., anonimato). Este só era apresentado após os profissionais concordarem com a participação no mesmo.

\section{Amostra}

Recolhemos dados junto de participantes a exercer as suas funções em todos os distritos portugueses e nos arquipélagos, sendo os distritos mais representados o do Porto (20\%), Braga (15\%), Lisboa (13\%) e Algarve (11\%).

A amostra total era composta por 610 profissionais, maioritariamente do sexo feminino (58\%), com idade média de 39,74 anos $(D P=9,51)$, variável entre 22 e 70 anos. Eram maioritariamente portugueses (99\%) e caucasianos (99\%). Trabalhavam na mesma função, em média, há 13,9 anos $(D P=9,5)$, variável entre um e 45 . Os participantes distribuíam-se pelas três áreas profissionais: $37 \%$ na área social, $33 \%$ na área da saúde e $30 \%$ OPC. Uma descrição detalhada da amostra, por área profissional, encontra-se na Tabela 1.

\section{Profissionais da área social}

O grupo de profissionais que trabalhava na área social era composto por 226 profissionais, maioritariamente do sexo feminino (85\%), com idade média de 40 anos (DP = $9,27)$ e com tempo médio na mesma função de 10,45 anos $(D P=8,42)$. Todos tinham habilitações académicas de nível superior, $71 \%$ licenciatura e $29 \%$ mestrado. Quanto à profissão, $29 \%$ eram psicólogos, $24 \%$ assistentes sociais, $19 \%$ educadores sociais, $6 \%$ animadores socioculturais e $4 \%$ juristas (a trabalhar em instituições de cariz social). Os restantes $18 \%$ foram agrupados em 'outras profissões', por 
Tabela 1 - Caracterização sociodemográfica dos participantes, por área profissional

\begin{tabular}{|c|c|c|c|c|c|c|}
\hline & \multicolumn{2}{|c|}{$\begin{array}{l}\text { Social } \\
(n=226)\end{array}$} & \multicolumn{2}{|c|}{$\begin{array}{l}\text { Saúde } \\
(n=203)\end{array}$} & \multicolumn{2}{|c|}{$\begin{array}{l}\text { Polícias } \\
(n=181)\end{array}$} \\
\hline & $\mathbf{n}$ & $\%$ & $\mathbf{n}$ & $\%$ & $\mathbf{n}$ & $\%$ \\
\hline \multicolumn{7}{|l|}{ Sexo } \\
\hline Feminino & 192 & 85 & 149 & 73 & 13 & 7 \\
\hline Masculino & 34 & 15 & 54 & 27 & 168 & 93 \\
\hline \multicolumn{7}{|l|}{ Etnia } \\
\hline Caucasiano/a & 222 & 98 & 202 & 99 & 181 & 100 \\
\hline Negro/a & 4 & 2 & 1 & 1 & - & - \\
\hline \multicolumn{7}{|l|}{ Nacionalidade } \\
\hline Portuguesa & 225 & 99 & 198 & 98 & 180 & 99 \\
\hline Estrangeira & 1 & 1 & 5 & 2 & 1 & 1 \\
\hline \multicolumn{7}{|c|}{ Habilitações Literárias } \\
\hline Secundário & - & - & - & - & 145 & 80 \\
\hline Licenciatura & 160 & 71 & 136 & 67 & 25 & 14 \\
\hline Mestrado & 66 & 29 & 60 & 30 & 11 & 6 \\
\hline \multirow[t]{2}{*}{ Doutoramento } & - & - & 7 & 3 & - & - \\
\hline & Média & $D P$ & Média & $D P$ & Média & $D P$ \\
\hline Idade & 40 & 9,27 & 40,95 & 11,16 & 38,04 & 7,36 \\
\hline Tempo de serviço & 10,45 & 8,42 & 16,33 & 10,90 & 15,48 & 7,16 \\
\hline
\end{tabular}

estarem pouco representadas nesta amostra (ex., antropólogos, sociólogos). No que respeita às instituições onde exerciam a sua profissão, $53 \%$ referiu trabalhar numa instituição pública/governamental; 23\% numa instituição de apoio à vítima; $15 \%$ numa Instituição Particular de Solidariedade Social (IPSS); $5 \%$ num Centro Local de Atendimento ao Imigrante (CLAII); 4\% referiu trabalhar numa instituição de acolhimento para vítimas de violência doméstica.

\section{Profissionais de saúde}

O grupo de profissionais que exercia a sua função na área da saúde era composto por 203 profissionais, maioritariamente do sexo feminino $(73 \%)$, com idade média de 40,95 anos $(D P=11,16)$ e com tempo médio na mesma função de 16,32 anos $(D P=10,9)$. Todos tinham habilitações académicas de nível superior, $67 \%$ tinham licenciatura, 30\% mestrado e 3\% doutoramento. Quanto à profissão, $55 \%$ eram médicos/as, $44 \%$ enfermeiros e $1 \%$ psicólogos, a trabalhar maioritariamente em ambiente hospitalar (71\%) ou centro de saúde (24\%). Os restantes trabalhavam em clínicas privadas (2\%), em IPSS (2\%) ou ONGs (1\%).

\section{Órgãos de polícia criminal}

O grupo dos OPC's era composto por 181 participantes, maioritariamente do sexo masculino (93\%), com idade média de 38,04 anos $(D P=7,36)$ e com tempo médio na mesma função de 15,48 anos $(D P=7,16)$. A maioria $(80 \%)$ tinha habilitações académicas de nível secundário, 14\% possuíam licenciatura e $6 \%$ mestrado.

\section{Experiência com populações multiculturais}

Quando questionados, 63\% dos participantes respondeu ter conhecimento de outras línguas, sendo que 35\% referiu falar mais do que uma língua estrangeira. As mais faladas pelos participantes eram Inglês (59\%), Francês (28\%) e Espanhol (24\%). Os restantes 37\% referiram não serem fluentes em nenhuma língua estrangeira.

No que respeita à experiência com populações multiculturais, a esmagadora maioria (91\%) dos profissionais já teve contacto com as mesmas. No questionário estavam listadas as populações imigrantes mais representadas em Portugal, de acordo com os dados estatísticos disponibilizados oficialmente no relatório Imigrantes, Fronteiras e Asilo (2014), com resposta aberta para a possibilidade de identificar outras populações não discriminadas. Assim, a maioria dos participantes já trabalhou com imigrantes oriundos da Europa de Leste (67\%), brasileiros (66\%), angolanos $(36 \%)$, cabo-verdianos $(32 \%)$ e, ainda, chineses (21\%). No que respeita a minorias étnicas, $15 \%$ já trabaIhou habitualmente com população cigana, de origem portuguesa e/ou estrangeira. A maioria dos profissionais (64\%) referiu ter trabalhado com mais do que uma população multicultural $(M=2,21 ; D P=1,42)$. Não existiam diferenças estatisticamente significativas a este nível, em função da 
área profissional, $F(2,607)=0,954, p=0,38$. Na Tabela 2 são apresentados os resultados por área profissional.

\section{RESULTADOS}

Perceção sobre a interação e a intervenção com populações multiculturais

De forma geral, os profissionais de ajuda de todas as áreas avaliaram-se como sendo bem-sucedidos na interação e intervenção junto de populações imigrantes e de minorias étnicas (Tabela 3). No entanto, foram encontradas diferenças significativas no que respeita ao sucesso percebido na interação e intervenção com algumas populações, sendo que os profissionais de saúde se avaliaram como mais competentes na interação e na intervenção com angolanos, cabo-verdianos e brasileiros, seguidos dos profissionais da área social e dos polícias. No que respeita à população chinesa, os profissionais de saúde avaliaram a sua capacidade de intervenção como significativamente mais positiva do que os polícias.

\section{Formação prévia em competência cultural}

A maioria dos participantes $(62 \%)$ referiu não ter tido qualquer contato formal com a temática da competência cultural. Os restantes $38 \%$ referiram que a mesma foi abordada em diferentes disciplinas na escola/universidade $(29 \%)$ e outros referiram ter assistido a uma aula que abordava especificamente a competência cultural (11\%). Contudo, essa temática foi sendo integrada ao longo da vida dos participantes de diferentes formas: $53 \%$ referiu ter adquirido conhecimento sobre o tema durante a sua experiência profissional ou tempo de serviço; $21 \%$ a partir de leituras sobre a temática por iniciativa própria; $19 \%$ participou num workshop onde a temática foi abordada; $11 \%$ tomou conhecimento através de profissionais de outras

Tabela 2 - Populações imigrantes e minorias étnicas atendidas, por área profissional

\begin{tabular}{|c|c|c|c|c|c|c|}
\hline \multirow[b]{2}{*}{ Populações imigrantes e minorias étnicas } & \multicolumn{2}{|c|}{$\begin{array}{c}\text { Social } \\
(n=226)\end{array}$} & \multicolumn{2}{|c|}{$\begin{array}{l}\text { Saúde } \\
(n=203)\end{array}$} & \multicolumn{2}{|c|}{$\begin{array}{l}\text { Polícias } \\
(n=181)\end{array}$} \\
\hline & $\mathbf{n}$ & $\%$ & $\mathbf{n}$ & $\%$ & $\mathbf{n}$ & $\%$ \\
\hline Angolanos & 92 & 41 & 77 & 38 & 48 & 27 \\
\hline Cabo-Verdianos & 91 & 40 & 53 & 26 & 49 & 27 \\
\hline Brasileiros & 152 & 67 & 131 & 65 & 121 & 67 \\
\hline Europa de Leste & 140 & 62 & 138 & 68 & 128 & 71 \\
\hline Chineses & 20 & 9 & 70 & 35 & 38 & 21 \\
\hline Ciganos & 33 & 15 & 32 & 16 & 23 & 13 \\
\hline
\end{tabular}

Tabela 3 - Perceção de sucesso na interação e intervenção com populações multiculturais, por área profissional

\begin{tabular}{|c|c|c|c|c|c|c|c|}
\hline & \multicolumn{2}{|c|}{$\begin{array}{l}\text { Social } \\
(n=226)\end{array}$} & \multicolumn{2}{|c|}{$\begin{array}{c}\text { Saúde } \\
(n=203)\end{array}$} & \multicolumn{2}{|c|}{$\begin{array}{l}\text { Polícias } \\
(n=181)\end{array}$} & \multirow[t]{2}{*}{$F$} \\
\hline & Média & $D P$ & Média & $D P$ & Média & $D P$ & \\
\hline \multicolumn{8}{|l|}{ Interação } \\
\hline Angolanos & 4,7 & 1,13 & 4,82 & 0,95 & 4,12 & 1,15 & $14,59^{* * *}$ \\
\hline Cabo-Verdianos & 4,78 & 1,16 & 5,1 & 1,09 & 4,31 & 1,17 & $19,42^{* * *}$ \\
\hline Brasileiros & 4,58 & 1,24 & 4,73 & 1,12 & 3,96 & 1,2 & 13,53 \\
\hline Europeus Leste & 3,48 & 1,39 & 3,28 & 1,3 & 3,36 & 1,42 & 0,44 \\
\hline Chineses & 4,14 & 1,39 & 4,11 & 1,14 & 3,67 & 1,29 & $6,69 * *$ \\
\hline Ciganos & 4,19 & 1,58 & 4,2 & 1,13 & 3,97 & 1,51 & 1,69 \\
\hline \multicolumn{8}{|l|}{ Intervenção } \\
\hline Angolanos & 4,29 & 1,15 & 4,71 & 0,96 & 4,02 & 1,19 & $12,03^{* * *}$ \\
\hline Cabo-Verdianos & 4,37 & 1,17 & 4,96 & 0,98 & 4,19 & 1,19 & $21,11^{* * *}$ \\
\hline Brasileiros & 4,24 & 1,20 & 4,69 & 1,07 & 3,86 & 1,24 & $13,55^{\star * *}$ \\
\hline Europeus Leste & 3,57 & 1,40 & 3,59 & 1,34 & 3,34 & 1,31 & 1,12 \\
\hline Chineses & 3,87 & 1,35 & 4,2 & 1,15 & 3,6 & 1,25 & $8,84^{* *}$ \\
\hline Ciganos & 3,84 & 1,43 & 3,9 & 1,47 & 2,94 & 1,47 & 2,73 \\
\hline
\end{tabular}


áreas, no seu local de trabalho; $3 \%$ tomou conhecimento através do seu supervisor institucional. Estes participantes tiveram contacto com a temática em mais do que um dos contextos descritos anteriormente, sendo o número médio de experiências formativas de 1,42 (DP = 1,32). Foram encontradas diferenças significativas ao nível das experiências formativas em competência cultural em função das três áreas profissionais, $F(2,607)=26,41, p<0,001$. 0 teste Post-Hoc de Gabriel revelou que os profissionais que exerciam na área social relataram significativamente mais experiências formativas em competência cultural $(M=1,82$, $D P=1,37)$, seguidos dos OPCs $(M=1,48, D P=1,34)$ e dos profissionais de saúde $(M=0,93, D P=1,1)$.

\section{Níveis percebidos de competência cultural}

De acordo com os resultados obtidos, as quatro dimensões que compõem a competência cultural encontram-se positivamente relacionadas (Tabela 4).

Os profissionais revelaram uma perceção positiva da sua competência cultural, com exceção do apoio organizacional. A média obtida para cada uma das dimensões foi de 3,61 $(D P=1,14)$ para a consciência cultural; 4,42 $(D P=$ $0,84)$ para o conhecimento cultural; 3,56 $(D P=1,42)$ para as aptidões técnicas; e 2,92 $(D P=1,12)$ para o apoio organizacional.

\section{Diferenças de sexo e competência cultural}

$\mathrm{Na}$ amostra total, existem diferenças significativas ao nível do conhecimento cultural em função do sexo, $t$ (608) $=4,68, p<0,001$, tendo sido as mulheres a percecionarem-se como mais competentes neste domínio. Os homens reportaram, de forma significativa, níveis mais elevados ao nível da consciência cultural, $t(608)=-4,33, p<0,001$.

Tabela 4 - Correlação entre dimensões da competência cultural

\begin{tabular}{|c|c|c|c|c|}
\hline Dimensões da competência cultural & 1 & 2 & 3 & 4 \\
\hline 1. Consciência cultural & - & & & \\
\hline 2. Conhecimento cultural & 0,26 & - & & \\
\hline 3. Aptidões técnicas & 0,48 & 0,32 & - & \\
\hline 4. Apoio organizacional & 0,35 & 0,41 & 0,28 & - \\
\hline
\end{tabular}

Todas as correlações são significativas a $p<0,01$

Tabela 5 - Níveis percebidos de competência cultural, por área profissional.

\begin{tabular}{|c|c|c|c|c|c|c|c|}
\hline & \multicolumn{2}{|c|}{$\begin{array}{c}\text { Social } \\
(n=226)\end{array}$} & \multicolumn{2}{|c|}{$\begin{array}{l}\text { Saúde } \\
(n=203)\end{array}$} & \multicolumn{2}{|c|}{$\begin{array}{l}\text { Polícias } \\
(n=181)\end{array}$} & \multirow[t]{2}{*}{$\boldsymbol{F}$} \\
\hline & Média & $D P$ & Média & $D P$ & Média & $D P$ & \\
\hline Consciência cultural & 3,21 & 1,18 & 3,8 & 0,97 & 3,9 & 1,13 & $24,32^{* * *}$ \\
\hline Conhecimento cultural & 4,69 & 0,74 & 4,32 & 0,83 & 4,19 & 0,87 & $21,43^{* * *}$ \\
\hline Aptidões técnicas & 3,16 & 1,19 & 3,86 & 0,97 & 3,7 & 1,12 & $23,81^{\text {*** }}$ \\
\hline Apoio organizacional & 3,33 & 1,11 & 2,44 & 0,99 & 2,94 & 1,07 & $37,97^{* * *}$ \\
\hline
\end{tabular}


Perceção de competência cultural por área profissional

Os resultados por grupo profissional são apresentados na Tabela 5.

Foi possível verificar a existência de diferenças estatisticamente significativas na pontuação obtida nas quatro dimensões da competência cultural. Os profissionais pontuaram, de forma geral, mais elevado ao nível do conhecimento cultural, seguindo-se a consciência cultural e as aptidões técnicas. Foram os profissionais da área social que referiram uma maior perceção ao nível do conhecimento cultural face aos outros grupos profissionais; na consciência cultural foram os OPCs que pontuaram mais alto; nas aptidões técnicas foram os profissionais de saúde que pontuaram mais alto. A dimensão apoio organizacional apresentou uma pontuação inferior em todos os grupos profissionais, sendo que na área da saúde os profissionais pontuaram significativamente abaixo das outras duas.

\section{DISCUSSÃO}

De forma geral, os profissionais avaliam-se de forma positiva no que respeita às diferentes componentes de competência cultural, sendo a dimensão do conhecimento cultural aquela onde foram obtidos scores mais elevados e a dimensão referente ao apoio organizacional a que obteve pontuações mais baixas. Isso poderá indicar que as instituições não estão ainda suficientemente capazes de se distanciar da cultura dominante e de adaptar o espaço e os materiais disponíveis à diversidade cultural das populações com as quais trabalham. ${ }^{50}$ Os profissionais inquiridos consideram-se bem-sucedidos sobretudo junto de minorias falantes de português (e.g., brasileiros, angolanos, cabo-verdianos), nomeadamente os profissionais de saúde. Comparativamente com o único estudo disponível realizado pelos autores do instrumento, ${ }^{5}$ o grupo dos terapeutas ocupacionais, nos EUA, apresentou, de uma forma global, uma perceção de competência cultural mais elevada do que os profissionais portugueses, quer da área social, quer da saúde e dos OPCs. De referir, no entanto, que os terapeutas ocupacionais que constituíam a amostra, para além de exercerem a sua prática num ambiente multicultural muito diferenciado da realidade portuguesa, eram, em média, mais velhos que os profissionais portugueses $(M=$ $43,3 ; D P=10,3)$ e possuíam mais anos de prática profissional $(M=17,32 ; D P=10,64)$.

Estudos anteriores demonstraram que os profissionais que trabalham com populações multiculturais tendem a percecionar-se como mais competentes culturalmente do que os que não têm esse tipo de experiência. ${ }^{5,51} \mathrm{Na}$ atual amostra, a grande maioria (91\%) trabalha habitualmente com, pelo menos, um grupo de pessoas de diferentes contextos culturais, sendo que uma maior diversidade de populações atendidas estava associada com uma melhor perceção de conhecimento cultural. Este resultado poderá estar relacionado com a aprendizagem empírica decorrente do contato com uma maior diversidade e familiaridade culturais, que permitirá um maior conhecimento sobre a sua história, os valores, as crenças e os comportamentos.
Pelo contrário, os profissionais que referiram uma maior diversidade de grupos atendidos relataram uma menor perceção de competência ao nível das aptidões práticas, o que poderá estar relacionado com uma exigência acrescida ao nível da especialização em diferentes contextos culturais, de forma a ajustar a prática profissional às necessidades de cada uma.

As profissionais mulheres revelaram um maior conhecimento cultural do que os homens, isto é, maior familiaridade com as características, a história, os valores, as crenças e os comportamentos de outras culturas. Os homens, por sua vez, percecionaram-se como tendo uma consciência cultural superior à das mulheres, ou seja, consideram estar mais capazes de fazer uma apreciação e de compreender a cultura de outras pessoas, reconhecimento dos próprios preconceitos em relação a outras culturas e exame crítico em relação a posições privilegiadas na sociedade. De notar que as áreas de saúde e social são mais homogéneas no que respeita à sua composição em função do sexo (73\% e $85 \%$, respetivamente), sendo que os OPCs são maioritariamente do sexo masculino (93\%). Este último resultado, apesar de contrariar outros estudos em que os homens apresentam ainda estereótipos e preconceitos em relação às vítimas, ${ }^{30}$ pode já ser o resultado de um grande investimento nacional que tem sido feito sobretudo na formação curricular e avançada das polícias em torno de crenças e estereótipos culturais. Por outro lado, os profissionais mais velhos revelaram maior conhecimento cultural, enquanto aqueles que exerciam há mais tempo a sua função relataram níveis mais elevados de aptidões práticas para intervir.

Aliados à experiência profissional, está o contacto prévio com a temática das competências culturais. Essas duas dimensões, de forma complementar, parecem fazer a diferença ao nível da competência cultural dos profissionais de ajuda (maior conhecimento, consciência cultural mais elevada, melhor apoio organizacional percebido), independentemente da área de atuação. Estes resultados são congruentes com estudos anteriores que referem que profissionais com mais experiências formativas e com mais experiência profissional tiveram mais oportunidades para refletir e contatar com populações culturalmente diferenciadas, ${ }^{5}$ inclusivamente na área da saúde, ${ }^{21}$ desenvolvendo assim a sua competência cultural.

Porém, de acordo com os dados obtidos, as experiências formativas e o contacto com a temática parecem insuficientes, na medida em que apenas $1 / 3$ dos profissionais reportou ter tido oportunidades estruturadas de formação nesse domínio. Efetivamente, a formação dos técnicos ao nível da competência cultural permite um maior conhecimento sobre os valores, crenças, hábitos e formas de interação dos grupos minoritários e é fundamental para que as diferenças culturais possam constituir um recurso para a intervenção. ${ }^{40}$

Numa análise mais minuciosa dos dados, os grupos de profissionais analisados diferem ao nível das suas perceções nas quatro dimensões da competência cultural. Os profissionais da área social consideram ter um maior conhe- 
cimento cultural, comparativamente com os profissionais das outras áreas. Este grupo de profissionais é também aquele que, no decorrer da sua carreira profissional, teve mais oportunidades formativas para desenvolver as suas competências neste domínio. Recorde-se que o número de experiências formativas revelou ser um fator preponderante ao nível do conhecimento cultural. Por outro lado, os profissionais de saúde diferenciam-se ao nível da perceção das aptidões técnicas, quando comparados com os outros grupos de profissionais. Este resultado poderá estar relacionado com o número de anos de prática profissional, fator que assumiu um papel preponderante também ao nível do desenvolvimento desta dimensão. De referir igualmente que foram os profissionais a trabalhar na área da saúde que avaliaram de forma mais negativa o apoio organizacional ao nível da competência cultural. Uma vez que todas as dimensões da competência cultural se encontram correlacionadas, um maior investimento neste domínio poderia significar também um incremento ao nível das aptidões e práticas profissionais. Este aspeto corrobora também a necessidade identificada pelo MIPEX ${ }^{49}$ na área da saúde, no que respeita à necessidade de investir na sensibilização dos prestadores de serviços de saúde, de forma a melhorar a capacidade de resposta às necessidades específicas de saúde dos migrantes a residir em Portugal, bem como o acesso ao serviço nacional de saúde.

\section{CONCLUSÃO}

Este estudo permitiu avaliar a perceção de competência cultural de profissionais de ajuda, oriundos de diferentes áreas, inserindo-se num projeto mais amplo que pretende avaliar o tipo e a qualidade das respostas dos serviços para as vítimas imigrantes a residir em Portugal. Estes dados corroboram outros que mostram, por um lado, que os comportamentos de procura de ajuda por parte das vítimas dependem da qualidade dos seus contactos com o sistema ${ }^{30}$ e, por outro, que as populações imigrantes poderão não procurar ajuda no país de acolhimento por terem a perceção da inexistência de serviços culturalmente competentes. ${ }^{52}$

Contudo, e tratando-se de um estudo exploratório, baseado numa recolha de dados online, os resultados não são passíveis de ser generalizados, uma vez que apesar de ter sido possível recolher dados em todos os distritos nacionais, territoriais e ilhas, a amostra não é representativa. O estudo permitiu avaliar a perceção dos profissionais acerca da sua competência cultural, não permitindo uma avaliação da sua prática efetiva, na eficácia do atendimento e intervenção junto de populações multiculturais.

Apesar das limitações descritas, este estudo permitiu realizar um conjunto de reflexões acerca da necessidade de investir no desenvolvimento da competência cultural dos profissionais de ajuda, em Portugal. Este investimento resultaria na inclusão desta temática nos curricula académicos, na integração destas questões ao nível formação profissional contínua e avançada, nas missões estratégicas dos serviços públicos e nas políticas institucionais. A realização de investigações futuras que permitam um maior conhecimento sobre o desenvolvimento da competência cultural dos profissionais de ajuda também é necessária e urgente.

Para além disso, envolver um maior número de profissionais neste tipo de estudos, de forma a ser capaz de representar a realidade nacional, é outra das necessidades futuras. É ainda urgente a sua complementaridade com estudos de cariz qualitativo que permita uma reflexão crítica sobre as dificuldades dos profissionais no atendimento a vítimas oriundas de culturas minoritárias, bem como sobre as lacunas existentes no desenvolvimento da competência cultural. Finalmente, um dos nossos próximos objetivos será o de complementar este estudo com investigações junto das populações multiculturais (e.g., vítimas de crime), no sentido de esclarecer se os profissionais de ajuda são, efetivamente, culturalmente competentes, desta vez na perspetiva dos utentes.

\section{PROTECÇÃO DE PESSOAS E ANIMAIS}

Os autores declaram que os procedimentos seguidos estavam de acordo com os regulamentos estabelecidos pelos responsáveis da Comissão de Investigação Clínica e Ética e de acordo com a Declaração de Helsínquia da Associação Médica Mundial.

\section{CONFIDENCIALIDADE DOS DADOS}

Os autores declaram ter seguido os protocolos do seu centro de trabalho acerca da publicação de dados.

\section{CONFLITOS DE INTERESSE}

Os autores declaram não terem qualquer conflito de interesse relativamente ao presente artigo.

\section{FONTES DE FINANCIAMENTO}

Os autores declaram não ter recebido subsídios ou bolsas para a elaboração do artigo.

\section{REFERÊNCIAS}

1. Crenshaw K, Shewchuk RM, Qu H, Staton LJ, Bigby JA, Houston TK, et al. What should we include in a cultural competence curriculum? An emerging formative evaluation process to foster curriculum development. Acad Med. 2011;86:333-41.

2. Gallegos JS, Tindall C, Gallegos SA. The need for advancement in the conceptualization of cultural competence. Adv Soc Work. 2008;9:51-62.

3. Muñoz JP. Culturally responsive caring in occupational therapy. Occup

Ther Int. 2007;14:256-80.

4. Sue DW. Multidimensional facets of cultural competence. Couns Psychol. 2001;29:790-821.

5. Balcazar FE, Suarez-Balcazar Y, Taylor-Ritzler T. Cultural competence: development of a conceptual framework. Disabil Rehabil. 2009;31:115360.

6. Shen Z. Cultural competence models and cultural competence 
assessment instruments in nursing: a literature review. J Transcult Nurs. 2015;26:308-21.

7. Suarez-Balcazar Y, Baicazar F, Taylor-Ritzier T, Portillo N, Rodakowsk J, Garcia-Ramirez M, et al. Development and validation of the cultural competence assessment instrument: A factorial analysis. J Rehabil. 2011;77:4-13.

8. Garneau AB, Pepin J. Cultural competence: a constructivist definition. J Transcult Nurs. 2015;26:9-15.

9. Darawsheh W, Chard G, Eklund M. The challenge of cultural competency in the multicultural 21st century: a conceptual model to guide occupational therapy practice. Open J Occup Ther. 2015;3:

10. Kleinman A, Benson P. Anthropology in the clinic: the problem of cultural competency and how to fix it. PLoS Med. 2006;3:e294.

11. Willen SS, Carpenter-Song E. Cultural competence in action: 'lifting the hood' on four case studies in medical education. Cult Med Psychiatry. 2013;37:241-52.

12. Baker K, Beagan B. Making assumptions, making space: an anthropological critique of cultural competency and its relevance to queer patients. Med Anthropol Q. 2014;28:578-98.

13. Carpenter-Song EA, Nordquest Schwallie M, Longhofer J. Cultural competence reexamined: critique and directions for the future. Psychiatr Serv. 2007;58:1362-5.

14. Cross T. Towards a culturally competent system of care: a monograph on effective services for minority children who are severely emotionally disturbed. Washington DC: CASSP Technical Assistance Center Georgetown University Child Development Center; 1989.

15. Gallegos JS. The ethnic competence model for social work education. In: White BW, editors. Color in a white society. Silver Spring: National Association of Social Workers; 1982.

16. Pedersen PB, Marsella AJ. The ethical crisis for cross-cultural counseling and therapy. Prof Psychol. 1982;13:492-500.

17. Sue DW, Bernier JE, Durran A, Feinberg L, Pedersen P, Smith EJ, et al. Position paper: cross-cultural counseling competencies. Couns Psychol. 1982;10:45-52.

18. Campinha-Bacote J. The process of cultural competence in the delivery of healthcare services: a model of care. J Transcult Nurs. 2002;13:1814.

19. Purnell M. Casting, replication, and anaglyph stereo imaging of microscopic detail in fossils, with examples from conodonts and other jawless vertebrates. Palaeontol Electron. 2003;6:1-11.

20. Shen Z. Cultural competence models in nursing: a selected annotated bibliography. J Transcult Nurs. 2004;15:317-22.

21. Sodowsky GR, Taffe RC, Gutkin TB, Wise SL. Development of the multicultural counseling Inventory: a self-report measure of multicultural competencies. J Couns Psychol. 1994;41:137-48.

22. Sue DW, Arredondo P, McDavis RJ. Multicultural counseling competencies and standards: a call to the profession. J Multicult Couns Devel. 1992;20:64-88.

23. Dean RAK. Cultural competence: nursing in a multicultural society. Nurs Womens Health. 2010;14:50-9.

24. Ingram RR. Using Campinha-Bacote's process of cultural competence model to examine the relationship between health literacy and cultural competence. J Adv Nurs. 2012;68:695-704.

25. Reimann JO, Talavera GA, Salmon M, Nuñez JA, Velasquez RJ. Cultural competence among physicians treating Mexican Americans who have diabetes: a structural model. Soc Sci Med. 2004;59:2195-205.

26. Brach C, Fraser I. Can cultural competency reduce racial and ethnic health disparities? A review and conceptual model. Med Care Res Rev. 2000;57:181-217.

27. Schouten BC, Meeuwesen L. Cultural differences in medical communication: a review of the literature. Patient Educ Couns. 2006;64:21-34

28. Butow PN, Bell ML, Aldridge LJ, Sze M, Eisenbruch M, Jefford M, et al. Unmet needs in immigrant cancer survivors: a cross-sectional population-based study. Support Care Cancer. 2013;21:2509-20.

29. Haj-Yahia MM, Uysal A. Beliefs about wife beating among medical students from Turkey. J Fam Violence. 2007;23:119-33.

30. Machado C, Matos M, Saavedra R, Cruz O, Antunes C, Pereira M, et al. Crenças e atitudes dos profissionais face à violência conjugal: estudos com profissionais de saúde, polícias e professores. Acta Med Port. 2009;22:735-42.

31. Narayanan G. Theorizing police response to domestic violence in the Singaporean context: police subculture revisited. J Crim Justice. 2005;33:429-39.

32. Tam SY, Tang CS. Comparing wife abuse perceptions between chinese police officers and social workers. J Fam Violence. 2005;20:29-38.

33. MenjÍVar C, Salcido O. Immigrant women and domestic violence: common experiences in different countries. Gend Soc. 2002;16:898920

34. Freedman J, Jamal B. Violence against migrant and refugee women in the Euromed region. Case studies: France, Egypt \& Marocco. Copenhagen: Euro-Mediterranean Human Rights Network; 2008.

35. Silva A, Azevedo L. Mulheres imigrantes e violência doméstica. In: Imigração e etnicidade - Vivências e trajetórias de mulheres em Portugal. Lisboa: SOS Racismo. 2005:139-47.

36. Tam D. Culturally responsive advocacy intervention with abused chinese-canadian women. Br J Soc Work. 2004;34:269-277.

37. Hassouneh-Phillips D. American muslim women's experiences of leaving abusive relationships. Health Care Women Int. 2001;22:415-32.

38. Shirwadkar S. Canadian domestic violence policy and Indian immigrant women. Violence Against Women. 2004;10:860-79.

39. Bui HN. Help-seeking behaviour among abused immigrant women. Violence Against Women. 2003;9:207-39.

40. Coelho C, Machado C. Competências culturais no atendimento a vítimas. In: Vitimologia: das novas abirdagens teóricas às novas práticas de intervenção. Braga: Psiquilíbios edições; 2010. p. 169-95.

41. Hyman I, Forte T, Mont J Du, Romans S, Cohen MM. Help-seeking rates for intimate partner violence (IPV) among Canadian immigrant women. Health Care Women Int. 2006;27:682-94.

42. Das Dasgupta S. Women's realities: Defining violence against women by immigration, race, and class. Issues Intimate Violence. 1998:209-19.

43. Gillum TL. Improving services to African American survivors of IPV: from the voices of recipients of culturally specific services. Violence Against Women. 2009;15:57-80.

44. Machado C, Dias AR. Abordagens culturais à vitimação: $O$ caso da violência conjugal. In: Machado $C$, editores. Vitimologia: das novas abordagens teóricas às novas práticas de intervenção. Braga: Psiquilíbios edições; 2010. p. 13-44.

45. Conde R, Gonçalves M, Matos M. Vítimas de violência conjugal: Intervenção multicultural com mulheres marginalizadas. In: Sani I, Caridade S, editores. Violência, agressão e vitimação: Práticas para a intervenção. Coimbra: Almedina; 2013. p. 105-26.

46. Pusseti C, Ferreira J, Lechner E, Santinho C. Migrantes e saúde mental - a construção da competência cultural. Lisboa: ACIDI; 2009.

47. Reis A. Cuidar de Imigrantes: das interações em contexto à construção de competências culturais nos enfermeiros. Rev Enferm. 2014;IV:61-9.

48. Presidência do Conselho de Ministros. Resolução do Conselho de Ministros n- ${ }^{0} 12-B$. Plano Estratégico nacional para as Migrações 20152020. Diário da República. I série. 2015;56:1654.

49. Migrant integration police index (MIPEX). Integration policies: Who benefits? The development and use of indicators in integration debates. 2015. [consultado 2015 out 10]. Disponível em: http://www.mipex.eu/ portugal.

50. Abney VD. Cultural competency in the field of child maltreatment. In: The APSAC Handbook on Child Maltreatment 2nd Ed. Thousand Oaks: Sage; 2002. p. 477-86.

51. Rogerson M, Emes C. Physical activity, older immigrants and cultural competence: A guide for fitness practitioners. Act Adapt Aging. 2006;30:15-28.

52. Kaukinen C, Demaris A. Sexual assault and current mental health: the role of help-seeking and police response. Violence Against Women. 2009;15:1331-57. 